\title{
Diversity of hysteresis in a fully cooperative coinfection model
}

Cite as: Chaos 28, 023107 (2018); https://doi.org/10.1063/1.4996807

Submitted: 19 July 2017 . Accepted: 28 December 2017 . Published Online: 06 February 2018

Jorge P. Rodríguez (D), Yu-Hao Liang, Yu-Jhe Huang, and Jonq Juang
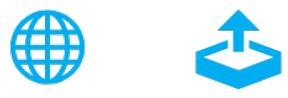

Export Citation

\section{ARTICLES YOU MAY BE INTERESTED IN}

Interactive social contagions and co-infections on complex networks

Chaos: An Interdisciplinary Journal of Nonlinear Science 28, 013120 (2018); https:// doi.org/10.1063/1.5010002

Ionic wave propagation and collision in an excitable circuit model of microtubules Chaos: An Interdisciplinary Journal of Nonlinear Science 28, 023106 (2018); https:// doi.org/10.1063/1.5001066

Complexity study on the Cournot-Bertrand mixed duopoly game model with market share preference

Chaos: An Interdisciplinary Journal of Nonlinear Science 28, 023101 (2018); https:// doi.org/10.1063/1.5001353

\section{AIP Author Services English Language Editing}




\title{
Diversity of hysteresis in a fully cooperative coinfection model
}

\author{
Jorge P. Rodríguez, ${ }^{1}$ Yu-Hao Liang, ${ }^{2}$ Yu-Jhe Huang, ${ }^{2}$ and Jonq Juang ${ }^{2, a)}$ \\ ${ }^{1}$ Instituto de Física Interdisciplinary Sistemas Complejos IFISC (CSIC-UIB), 07122 Palma de Mallorca, Spain \\ ${ }^{2}$ Department of Applied Mathematics, National Chiao Tung University, 300 Hsinchu, Taiwan
}

(Received 19 July 2017; accepted 28 December 2017; published online 6 February 2018)

\begin{abstract}
We propose a fully cooperative coinfection model in which singly infected individuals are more likely to acquire a second disease than susceptible ones and doubly infected individuals are also assumed to be more contagious than singly infected ones. The dynamics of such a fully cooperative coinfection model is investigated through the well-mixed approach. In particular, discontinuous outbreak transitions from the disease free state or the low prevalence state to the high prevalence state can be separately observed as a disease transmission rate crosses a threshold $\alpha_{o}$ from the below when the epidemic is still in the early stages. Moreover, discontinuous eradications from the high prevalence state to the low prevalence or disease free state are also separately seen as the transmission rate reaches a threshold $\alpha_{e}\left(<\alpha_{0}\right)$ from the above when the outbreak occurs. Such phenomena constitute three types of hysteresis, where only one type has been identified before. Complete characterization of these three types of hysteresis in terms of parameters measuring the uniformity of the model is both analytically and numerically provided. Published by AIP Publishing.

https://doi.org/10.1063/1.4996807
\end{abstract}

Characterizing the behavior of multi-disease or multistrain models is important to address some relevant issues in biology and ecology. This topic is becoming more and more relevant for the community of infectious disease modellers, mathematical biologists, and complex system scholars in general. In this work, we study the dynamics of an epidemic model of two interacting diseases where infection status is not mutually exclusive and spread of both diseases is enhanced when they are infecting a single individual. In particular, the model can undergo three types of hysteresis, indicating the discontinuous phase transitions from the disease-free state or the low prevalence state to the high prevalence state and from the high prevalence state to the low prevalence state or the disease free state. Such phenomena are sharply in contrast to a single disease model which only exhibits a continuous transition. Moreover, the results also highlight the importance of preventing the outbreak of disease since it would then take greater effort to eradicate it.

\section{INTRODUCTION}

Mathematical modelling has become a fundamental tool to understand the basic features of disease spreading in a group. ${ }^{1,2}$ We can deal with this problem with two approaches: considering a well-mixed system and approximating it with an interaction all-to-all, i.e., the mean-field approximation, or taking into account a topology that specifies who interacts with whom. Hence, the theory of both dynamic systems and complex networks $^{2-4}$ is fundamental for this kind of study. Two main compartmental models have been proposed for the dynamics of a spreading disease: one, considering three kinds

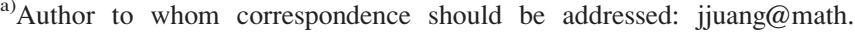
nctu.edu.tw
}

of individuals, where susceptibles $(\mathrm{S})$, when exposed to the disease, become infective (I) and afterwards become recovered $(\mathrm{R})$, getting immunized against the disease, is known as the SIR model ${ }^{5}$ the other model, known as the SIS model, ${ }^{6}$ represents diseases that are spread by pathogens that frequently mutate such that individuals do not get immunized but come back to the susceptible state after the infective phase. In fact, those models have been proposed not only in the context of disease spreading but also for other spreading processes, such as computer viruses epidemics. ${ }^{7}$

Most studies on disease spreading focus on one disease infecting a population. ${ }^{8-10}$ However, different pathogens may interact with each other on the host. For instance, there are cases in which infection with one disease strengthens a host's immunity against other infection. ${ }^{1-17}$ In those competitive systems, regimes such as extinction, coexistence, and one strain dominance have been identified. On the other hand, it has also been reported that people with human immunodeficiency virus (HIV) infections are more likely to get other infections such as hepatitis or tuberculosis. ${ }^{18-21}$ Such cooperative disease spreading models have recently been studied in Refs. 22-27. Interesting dynamics, like the appearance of abrupt transitions, in contrast to the typical second order phase transition found in the single disease spreading model, have been reported. ${ }^{24-26}$ Specifically, for SIS dynamics, there is a hysteresis behavior: In the early stages of diseases spreading when the infected population is small, the models exhibit discontinuous outbreak transition from the disease free state to the high prevalence state as a transmission rate $\alpha$ crosses a threshold $\alpha_{o}$ from the below, while in the reverse scenario, that is, when the outbreak of the diseases occurs, they exhibit discontinuous eradication transition from the high prevalence state to the disease free state as the transmission rate $\alpha$ reaches a threshold $\alpha_{e}\left(<\alpha_{0}\right)$ from the above. The above described phase transition is to be called a hysteresis of $C$ type 


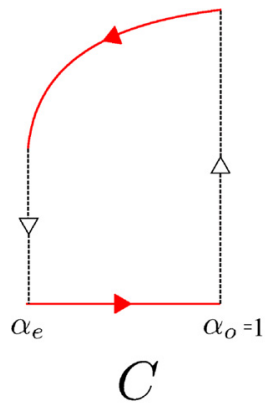

(a)

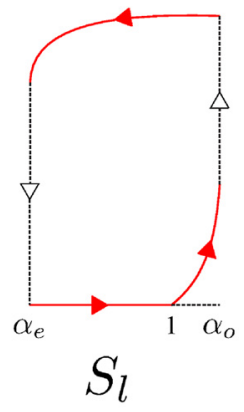

(b) (c)

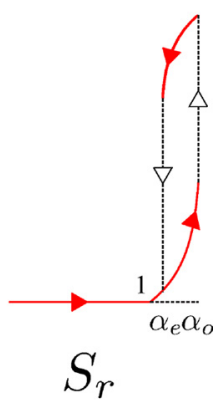

FIG. 1. Three types of hysteresis loops. The horizontal axis represents the homogeneous transmission rate $\alpha$ for a susceptible individual getting either one of the diseases. The vertical axis indicates the fraction of infected population. The horizontal level corresponds to no infected population. Our model has two stable fixed points whenever $\alpha_{e} \leq \alpha \leq \alpha_{o}$. Consequently, for $\alpha_{e} \leq \alpha \leq \alpha_{o}$, it would yield two stable epidemic state curves, indicating the infected population as represented by the red solid curves above. In the early stages of epidemics (resp., When the outbreak of the diseases occurs), as $\alpha$ gradually increases (resp., decreases) to $\alpha_{o}$ (resp., $\alpha_{e}$ ), the infected population has a discontinuous jump (resp., drop) as indicated by the black dotted lines. By choosing suitable parameters of our model, there exist three types of hysteresis loops as demonstrated above.

[Fig. 1(a)]. The difference in $\alpha_{e}$ and $\alpha_{o}$ points out that once the outbreak of the diseases occurs, driving down the transmission rate to $\alpha_{o}$ is not enough to eradicate the diseases. In fact, we need to drive the rate further down to $\alpha_{e}$ for the epidemic to die out, which requires more effort and results in greater economic costs. Note that in the cooperative coinfection models considered in Refs. 22, and 24-26, it was only assumed that a singly infected individual is more likely to get infected with a second disease than a susceptible one.

In this work, we consider a fully cooperative coinfection model for two diseases, in which we further assume that a doubly infected individual is more contagious than a singly infected one. Our model has two stable fixed points for $\alpha_{e}<\alpha<\alpha_{o}$, where $\alpha_{e}$ and $\alpha_{o}$ depend on other parameters of the model. Moreover, it has a unique stable fixed point whenever $\alpha<\alpha_{e}$ or $\alpha>\alpha_{o}$. Specifically, to form a hysteresis of $C$ type, the corresponding stable fixed points are the high prevalence state and the disease free state for $\alpha_{e} \leq \alpha \leq \alpha_{o}=1$. To form a hysteresis of $S_{l}$ type, we need to have a stable disease free state and a high prevalence state for $\alpha_{e} \leq \alpha \leq 1$ and two stable endemic fixed points, i.e., high and low prevalence states, for $1 \leq \alpha \leq \alpha_{o}$. To have a hysteresis of $S_{r}$ type, we need to have two stable endemic fixed points for $\alpha_{e} \leq \alpha \leq \alpha_{o}$, where $\alpha_{e}>1$. The last two types of hysteresis are to be further explained. Suppose that the model has discontinuous outbreak [resp. (To save space, from now on we shall write resp. as the abbreviation of respectively), eradication] transition from the low (resp., high) prevalence state to the high prevalence (resp., disease free) state as the transmission rate crosses a threshold $\alpha_{o}$ (resp., $\alpha_{e}$ ) from the below (resp., above). Then, the above described phase transition is called a hysteresis of $S_{l}$ type [Fig. 1(b)]. Moreover, suppose that a discontinuous outbreak (resp., eradication) transition from the low (resp., high) prevalence state to the high (resp., low) prevalence state can be observed as the transmission rate reaches a threshold $\alpha_{o}$ (resp., $\alpha_{e}$ ) from the below (resp., above). Then, the above mentioned phase transition is called a hysteresis of $S_{r}$ type [Fig. 1(c)]. For the single disease model, there exists only one stable fixed point. Hence, the phase transition is continuous. For the partially cooperative coinfection model such as those considered in Refs. 22 and 24-26, only the hysteresis of type $C$ has been identified.

The organization of this paper is as follows. In Sec. II, the well-mixed approach for our fully cooperative coinfection model is introduced and analytically studied. Numerical simulation results to illustrate our main results are also provided. The effect of heterogeneity in two diseases on the corresponding model is numerically investigated in Sec. III. The summary of the main results and the concluding remarks on the future work are given in Sec. IV. All proofs of our results are given in the Appendix.

\section{WELL-MIXED SYSTEM: HOMOGENEOUS CASE}

We study a well-mixed particle system for the fully cooperative coinfection dynamics with two diseases, A and B. We define $S, I_{A}, I_{B}$, and $I_{A B}$ as the fractions of susceptible individuals and the ones infected with diseases $\mathrm{A}, \mathrm{B}$, and $\mathrm{AB}$ in the population, respectively. Susceptible individuals get a primary infection from a singly infected individual carrying disease $\mathrm{A}$ or $\mathrm{B}$ with a homogenous rate $\alpha$, bringing the total rate of transmission of susceptible class to $2 \alpha$. Singly infected individuals with either disease A or B obtain another infection from a doubly infected individual with a higher homogeneous rate $\beta(>\alpha)$, taking the total rate of transmission of the singly infected class to $2 \beta$. It should be mentioned that simultaneous recovery from both diseases and simultaneous infection by both diseases are not considered. Moreover, the cooperation makes the doubly infected individuals more virulent in a way that they infect susceptibles (resp. singly infected) with a rate $a \alpha, a \geq 1$ (resp. $b \beta, b \geq 1$ ). Then, the corresponding mean field equations for this dynamic system are

$$
\begin{gathered}
\dot{S}(t)=-\alpha S\left(I_{A}+a I_{A B}\right)-\alpha S\left(I_{B}+a I_{A B}\right)+I_{A}+I_{B}, \\
\dot{I}_{A}(t)=\alpha S\left(I_{A}+a I_{A B}\right)-\beta I_{A}\left(I_{B}+b I_{A B}\right)+I_{A B}-I_{A}, \\
\dot{I}_{B}(t)=\alpha S\left(I_{B}+a I_{A B}\right)-\beta I_{B}\left(I_{A}+b I_{A B}\right)+I_{A B}-I_{B}, \\
\dot{I}_{A B}(t)=\beta I_{A}\left(I_{B}+b I_{A B}\right)+\beta I_{B}\left(I_{A}+b I_{A B}\right)-2 I_{A B} .
\end{gathered}
$$

Here, the homogeneous recovery rates for diseases A and B, without loss of generality, are set equal to the timescale, while the basic reaction scheme underlying the dynamic process is given in Table I. More precisely, $\alpha$ and $a \alpha$ (resp., $\beta$ and $b \beta$ ) are infection rates for the susceptible class (resp., singly infected class) who get infected by singly infected and doubly infected classes (resp., the complementarily infected class and doubly infected class), respectively. The quantity $a$ (resp., $b$ ) is the ratio between the infection rates of the doubly infected class and the singly infected class for the infecting susceptible class (resp., singly infected class). The larger the $a$ and $b$ values are, the more contagious the doubly infected class $\mathrm{AB}$ is. We call $a$ and $b$ the relative coinfection factors for the susceptible class and the singly infected class, respectively. The quantity $c:=\beta / \alpha$, which is termed the 
TABLE I. Basic reaction scheme for the disease spreading in the wellmixed model (1) containing five parameters $\alpha, \beta, a, b$, and $c$. The quantities $a$ and $b$ are the relative coinfection factors for the susceptible class and singly infected class, respectively. The quantity $c$ is the relative infection factor. When $a=b=c=1$, the model is said to be uniform and corresponds to the noninteracting disease spreading model. The larger the deviation of these quantities from 1 , the more nonuniform the model.

\begin{tabular}{lc}
\hline \hline Transmission & Rate \\
\hline$S \stackrel{A}{\rightarrow} A$ & $\alpha$ \\
$S \stackrel{B}{\rightarrow} B$ & $\alpha$ \\
$S \stackrel{A B}{\rightarrow} A$ & $a \alpha$ \\
$S \stackrel{A B}{\rightarrow} B$ & $a \alpha$ \\
$A \stackrel{B}{\rightarrow} A B$ & $\beta(=c \alpha)$ \\
$B \stackrel{A}{\rightarrow} A B$ & $\beta(=c \alpha)$ \\
$A \stackrel{A B}{\rightarrow} A B$ & $b \beta(=b c \alpha)$ \\
$B \stackrel{A B}{\rightarrow} A B$ & $b \beta(=b c \alpha)$ \\
\hline \hline
\end{tabular}

relative infection factor, is the ratio of the infection rates for the singly infected class and the susceptible class. The larger the $c$ value is, the easier the singly infected class gets infected with a secondary infection. Also note that the larger the quantities $a, b$ and $c$ deviate from 1 , the more nonuniform the model is. The case that $\min \{a, b, c\}>1$ (resp., $\max \{a, b, c\}<1$ ) corresponds to a fully cooperative (resp., competitive) coinfection model. If $\min \{a, b, c\}<1<\max \{a, b, c\}$, it is called a mixed type. In Ref. 26, the authors consider a partially cooperative model, i.e., $a, b=1$ and $c>1$, where the hysteresis of type $C$ was found, provided that $c$ is sufficiently large.

The dynamics of model (1) is depicted by its fixed points. Let $E^{*}:=\left(S^{*}, I_{A}^{*}, I_{B}^{*}, I_{A B}^{*}\right)$ be a fixed point of (1). Then, $E^{*}$ is a nontrivial endemic fixed point, i.e., $\left(I_{A}^{*}\right)\left(I_{B}^{*}\right)$ $\left(I_{A B}^{*}\right) \neq 0$, if and only if $I_{A}^{*}=I_{B}^{*}$ (Proposition A. 1 in the Appendix). To study the most interesting dynamics of the model, we consider its nontrivial endemic fixed point. Some direct computation yields that such fixed point $E^{*}$ satisfies the following:

$$
\begin{aligned}
I_{A}^{*} & =I_{B}^{*}=\frac{a \alpha S^{*}}{1+(2 a-1) \alpha S^{*}}\left(1-S^{*}\right), \\
I_{A B}^{*} & =\frac{1-\alpha S^{*}}{1+(2 a-1) \alpha S^{*}}\left(1-S^{*}\right),
\end{aligned}
$$

where $S^{*}$, satisfying

$$
0 \leq S^{*}<1 \quad \text { and } \quad 0 \leq \alpha S^{*} \leq 1,
$$

is a solution of

$$
\begin{aligned}
f(S, \alpha):= & a(b-a) c \alpha^{3} S^{3} \\
& +[\alpha a(a-b) c-a b c+2 a-1] \alpha^{2} S^{2} \\
& +(\alpha a b c-2 a+2) \alpha S-1=0 .
\end{aligned}
$$

For any $a, b \geq 1$ and $c>0$, Eq. (3) defines a solution curve $(S, \bar{\alpha}(S)), 0<S \leq 1$, satisfying $f(S, \bar{\alpha}(S))=0$. In particular, for any fixed $a, b \geq 1, c>0$, and $0<S \leq 1$, there exists a unique positive $\bar{\alpha}(=\bar{\alpha}(S))$ on $(0,1 / S]$ such that $f(S, \bar{\alpha}(S))=0$ (see Proposition A.2 in the Appendix). The graph of the function $\bar{\alpha}(S), 0<S \leq 1$ is to be studied next. We address it by making the change in the variable

$$
T=\alpha S .
$$

Clearly, by (3)

$$
\begin{aligned}
\bar{\alpha}(S) & =\frac{a(a-b) c T^{3}+(a b c-2 a+1) T^{2}+2(a-1) T+1}{a c T[(a-b) T+b]} \\
& =: \tilde{\alpha}(T) .
\end{aligned}
$$

Here, $0<T \leq 1$. Using Proposition A.3 in the Appendix, we have that the monotonicity structure of functions $\bar{\alpha}(S)$ and $\tilde{\alpha}(T)$ on the interval $(0,1]$ is identical. Hence, to study the dependence of the nontrivial endemic fixed points on the parameters, it suffices to graph the function of $\tilde{\alpha}(T)$, which is described in Theorem 2.1. The detailed analysis of the graph of $\tilde{\alpha}(T)$ is provided in Proposition A.4 - A.7 in the Appendix. The stability of fixed points of system (1), stated in Theorem 2.2, is also supplied. Its proof is given at the end of the Appendix.

Theorem 2.1. For parameters $a, b \geq 1, \lim _{T \rightarrow 0^{+}} \tilde{\alpha}(T)=\infty$ and $\tilde{\alpha}(1)=1$. Define $c_{2}\left(=c_{2}(a)\right)$ by

$$
c_{2}=\frac{2}{a} \text {. }
$$

Then, the following assertions (I) and (II) hold.

(I) $\quad$ See Figs. 2(a) and 3(a)] For $b \leq b_{c}(:=a+1 / 2)$, $\tilde{\alpha}(T)$ is concave upward on $(0,1]$. Moreover, the following two assertions hold.

(a)

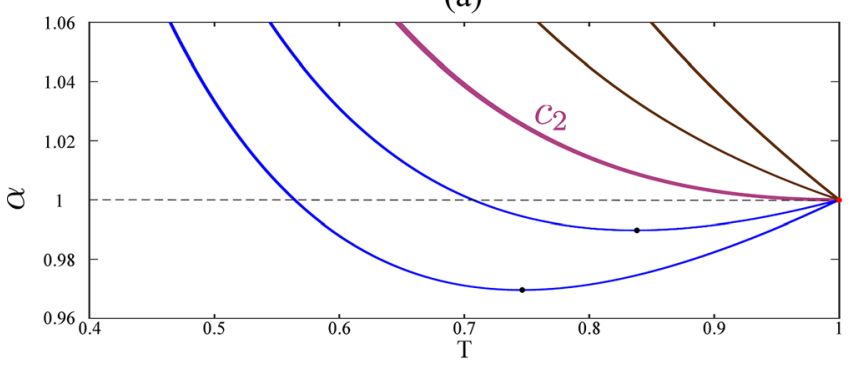

(b)

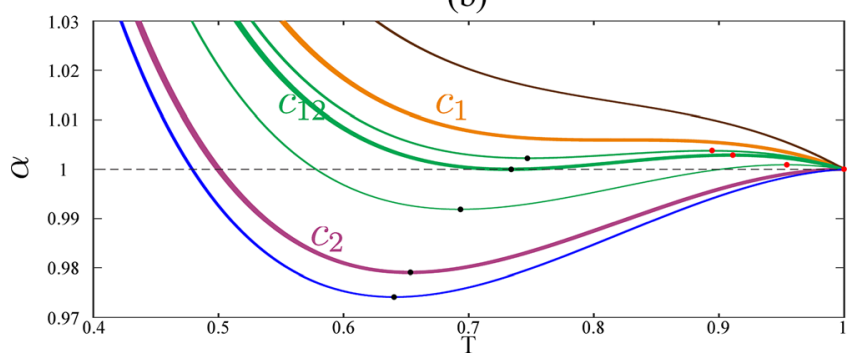

FIG. 2. Graphs of $\tilde{\alpha}(T)$ with $a=1, b=1.25$ or 2 , and various $c>0$. The $\alpha$ coordinates of local minimum and maximum of $\tilde{\alpha}(T)$ are $\alpha_{e}$ and $\alpha_{o}$, respectively. We color all the local minima (resp., maxima) by black (resp., red). (a) The numerically produced graphs of $\tilde{\alpha}(T)$ are consistent with the assertions in Theorem 2.1(I). Here, the parameters are set to be $(a, b)=(1,1.25)$ and $c$ varies discretely from $1.5<1.75<c_{2}(1)(=2)<2.25<2.5$, which correspond to the five curves in the panel from the right to the left, respectively. At $c=c_{2}, \tilde{\alpha}(T)$ is tangent to $\alpha=1$ at $T=1$. (b) The numerically produced graphs of $\tilde{\alpha}(T)$ are consistent with the assertions in Theorem 2.1(II). Here, the parameters are set to be $(a, b)=(1,2)$ and $c$ varies discretely from $1.75<c_{1}(1,2)(\approx 1.821)<1.85<c_{12}(1,2)(\approx 1.866)<1.92<c_{2}(1)(=2)$ $<2.03$, which correspond to the seven curves in the panel from the right to the left, respectively. At $c=c_{1}$, the tangent to $\tilde{\alpha}(T)$ at the unique inflection point $\gamma$ is horizontal, while at $c=c_{12}, \tilde{\alpha}(T)$ is tangent to $\alpha=1$ at some $T_{*}$ on $(0,1)$. Here, $\tilde{\alpha}\left(T^{*}\right)=\alpha_{e}$. 
(a)

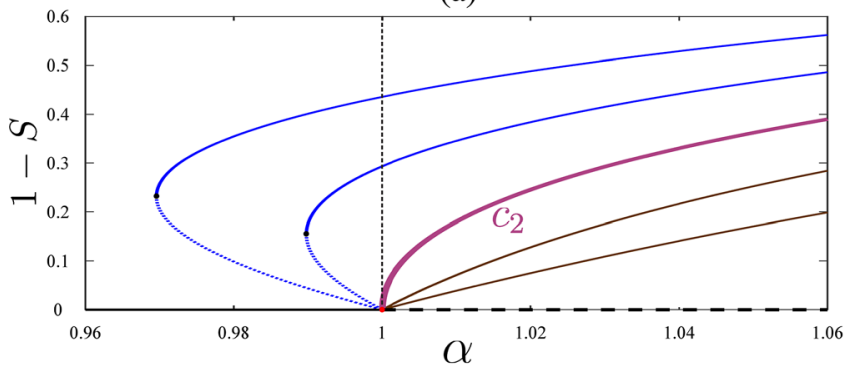

(b)

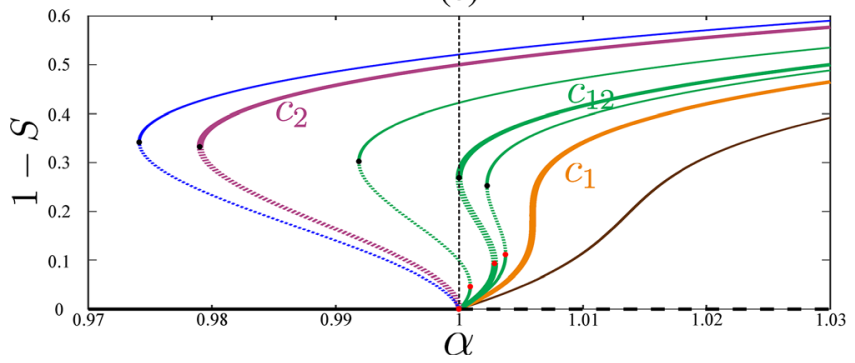

FIG. 3. Graphs of endemic state curve $\tilde{S}$ on the $(\alpha, 1-S)$ plane. The parameter set chosen to generate the curves displayed in (a) and (b) is the same as chosen from Figs. 2(a) and 2(b), respectively. The solid (resp., dotted) part of curves indicates the stability (resp., instability) of endemic states or the disease free state. The two intersection points of dotted and solid lines are outbreak transmission rate $\alpha_{o}$, colored by red, and eradication transition rate $\alpha_{e}$, colored by black, with $\alpha_{e}<\alpha_{o}$. (a) Parameters $a, b$, and $c$ are chosen as those in Fig. 2(a), in which the relative coinfection factor $b(=1.25)$ does not deviate from $a(=1)$ by too much, i.e., $b \leq b_{c}=1.5$. The shape of corresponding curves $\tilde{S}$ changes from increasing to $C$ shaped as the relative infection factor $c$ increases. (b) Parameters $a, b$, and $c$ are chosen as those in Fig. 2(b). Here, $b>b_{c}=1.5$. The shape of corresponding curves $\tilde{S}$ undergoes more complicated transition as $c$ gradually increases. As seen in the panel, $\tilde{S}$ changes from increasing to $S_{r}$ shaped to $S_{l}$ shaped to $C$ shaped.

(I-i) For $c \leq c_{2}, \tilde{\alpha}(T)$ is concave upward and strictly decreasing on $(0,1]$. In particular, if $c$ $=c_{2}$, then $\tilde{\alpha}(T)$ is tangent to $\alpha=1$ at $T=1$. Moreover, if we define the endemic state curve $\tilde{S}$ to be the corresponding curve of $\tilde{\alpha}(T)$ graphed on the $(\alpha, 1-S)$ plane, then $\tilde{S}$ also strictly increases.

(I-ii) For $c>c_{2}, \tilde{\alpha}(T)$ has exactly one critical point at some point $T_{*}$ on $(0,1)$, which corresponds to a local minimum $\alpha_{e}\left(=\alpha_{e}(c)\right)$. Moreover, $\alpha_{e}<\tilde{\alpha}(1)(=1)$. Consequently, the corresponding $\tilde{S}$ on the $(\alpha, 1-S)$ plane is $C$ shaped.

(II) [See Figs. 2(b) and 3(b)] For $b>b_{c}, \tilde{\alpha}(T)$ has exactly one inflection point $\gamma$ on $(0,1)$. Define $c_{1}\left(=c_{1}(a, b)\right)$ and $c_{12}\left(=c_{12}(a, b)\right)$, respectively, by

$$
\begin{gathered}
c_{1}=\frac{\left[a^{2 / 3}(2 b-1)^{1 / 3}+(b-a)^{2 / 3}\right]^{3}}{a b^{3}}, \\
c_{12}=\frac{(2 a b-2 a+b)+2 \sqrt{a(2 b-1)(b-a)}}{a b^{2}} .
\end{gathered}
$$

Here, $c_{1}$ and $c_{12}$ satisfy the following properties, respectively. The slope of the tangent of the corresponding curve $\tilde{\alpha}(T)$ at the unique inflection point $\gamma$ is zero, and the corresponding curve $\tilde{\alpha}(T)$ is tangent to $\alpha=1$ at $T_{*}$, where $\tilde{\alpha}\left(T^{*}\right)=\alpha_{e}$. Then, the following three assertions hold.

(II-i) For $c \leq c_{1}, \tilde{\alpha}(T)$ strictly decreases on $(0,1]$. Consequently, the corresponding $\tilde{S}$ on the $(\alpha, 1-S)$ plane strictly increases.

(II-ii) For $c_{1}<c<c_{2}, \tilde{\alpha}(T)$ has a unique local minimum $\alpha_{e}\left(=\alpha_{e}(c)\right)$ and maximum $\alpha_{o}\left(=\alpha_{o}(c)\right)$ at $T_{*}$ and $T^{*}$, respectively, on $(0,1)$ with $T_{*}<T^{*}$ such that $\tilde{\alpha}(T)$ strictly decreases on $\left(0, T_{*}\right]$ $\cup\left[T^{*}, 1\right]$ while strictly increasing on $\left(T_{*}, T^{*}\right)$. Moreover

$$
\alpha_{e}(c) \begin{cases}>1 & \text { if } c<c_{12}, \\ =1 & \text { if } c=c_{12}, \\ <1 & \text { if } c>c_{12} .\end{cases}
$$

Consequently, the corresponding $\tilde{S}$ on the $(\alpha, 1-S)$ plane is $S$ shaped.

(II-iii) For $c>c_{2}, \tilde{\alpha}(T)$ has exactly one critical point at some point $T_{*}$ on $(0,1)$, which corresponds to a local minimum $\alpha_{e}\left(=\alpha_{e}(c)\right)$. Moreover, $\alpha_{e}<\tilde{\alpha}(1)(=1)$. Consequently, the corresponding $\tilde{S}$ on the $(\alpha, 1-S)$ plane is $C$ shaped.

Mathematically speaking, if $b \leq b_{c}$, then $\tilde{\alpha}(T)$ is always concave upward regardless of the values of $c$ [Theorem 2.1(I) and Fig. 2(a)]. Hence, as $c$ becomes larger, $\tilde{\alpha}(T)$ can only change from increasing to having a local minimum [Fig. 2(a)]. The critical value for the change in the structure of the graph occurs at $c=c_{2}$ when $\tilde{\alpha}(T)$ is tangent to $\alpha=1$ at $T=1$. However, if $b>b_{c}$, then $\tilde{\alpha}(T)$ always has a unique inflection point on $(0,1)$ [Theorem 2.1(II) and Fig. 2(b)]. The structure of the graph of $\tilde{\alpha}(T)$ becomes more complicated as one gradually increases $c$ [Fig. 2(b)]. Specifically, the graph of $\tilde{\alpha}(T)$ changes from increasing to having two local extrema to one local minimum as $c$ gradually increases. Such a structure change occurs at $c=c_{1}$ and $c=c_{2}$ when the tangent to $\tilde{\alpha}(T)$ at the unique infection $\gamma$ is horizontal and when $\tilde{\alpha}(T)$ is tangent to $\alpha=1$, respectively [Fig. 2(b)]. For $c_{1}<c<c_{12}$, the graph of the corresponding $\tilde{S}$ can be further distinguished into two types. In particular, we shall term the corresponding $\tilde{S}$ for $c \in\left(c_{1}, c_{12}\right)$ and $c \in\left(c_{12}, c_{2}\right) S_{r}$ and $S_{l}$ shaped, respectively. Such a classification is needed since $C$ shaped, $S_{r}$ shaped, and $S_{l}$ shaped all have different dynamics.

Theorem 2.2. For parameters $a, b \geq 1$, the stability of fixed points of (1) is given as follows:

(i) The disease free state is locally stable (resp., unstable) provided that $\alpha \leq 1$ (resp., $\alpha>1$ ).

(ii) The trivial endemic fixed points, i.e., $E^{*}=\left(\frac{1}{\alpha}, 1-\frac{1}{\alpha}\right.$, $0,0)$ or $\left(\frac{1}{\alpha}, 0,1-\frac{1}{\alpha}, 0\right)$, are unstable for all $\alpha>1$.

(iii) If $E^{*}$, a nontrivial endemic fixed point, corresponds to the point on the increasing (resp., decreasing) part of endemic state curve $\tilde{S}$ on the $(\alpha, 1-S)$ plane, then it is locally stable (resp., unstable).

With the help of the stability result stated in Theorem 2.2, those three shapes of the graph as illustrated in Fig. 3 
would yield three types of hysteresis loops as demonstrated in Fig. 1. In view of Theorem 2.2(iii), we need to plot Eqs. (6)-(8) to completely characterize the stabilities of $\tilde{S}$. To this end, we graph $c_{1}(a, b), c_{12}(a, b)$, and $c_{2}(a)$ with either $a$ or $b$ being fixed in Fig. 4. Specifically, $a$ (resp., $b$ ) is fixed to be 1 (resp., 3) in Fig. 4(a) [resp., Fig. 4(b)]. Both the (b, $c)$ and (a, $c)$ planes are divided into four parts, strictly increasing, $C$ shaped, $S_{l}$ shaped, and $S_{r}$ shaped, according to the results stated in Theorem 2.1(I) and (II). Furthermore, it then follows from Theorem 2.2 that the dynamics of the model with parameters chosen from those four parts can also be completely understood. Specifically, those four parts indicated above would yield the following dynamics: a continuous transition, hysteresis loops of $C$ type, $S_{l}$ type, and $S_{r}$ type, respectively. Note that $a, b$, and $c$ are the measurement for the degree of uniformity in the coinfection model. The assumptions that $1 \leq b \leq b_{c}$ and $c \leq c_{2}$ or $b>b_{c}$ and $c \leq$ $c_{1}$ indicate that the model under consideration is relatively uniform. Such a model exhibits a continuous transition at $\alpha$ $=1$, the same threshold as the single disease spreading according to the SIS model. In short, for such a system, it acts as non-interacting. If the relative infection factor $c$ is large, more precisely $c>c_{2}$, then the model exhibits the hysteresis of $C$ type regardless of what relative coinfection factors $a$ and $b$ are. If the difference in relative coinfection factors $a$ and $b$ is large, i.e., $b>b_{c}$, then hysteresis behavior will occur with smaller $c$ as compared to the case that $b \leq b_{c}$. In particular, if $c$ is between $c_{1}$ and $c_{2}$, then

(a)

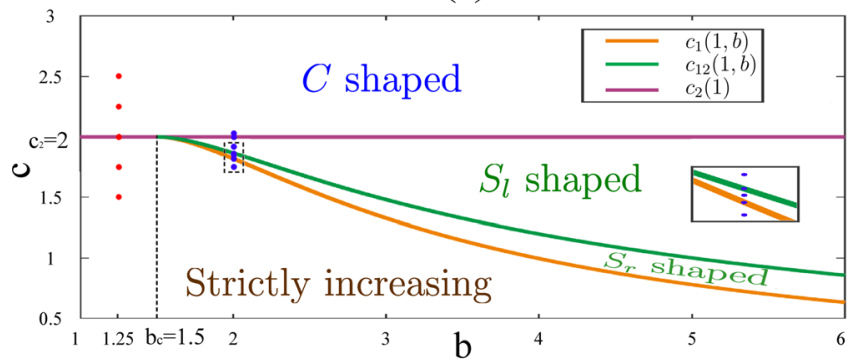

(b)

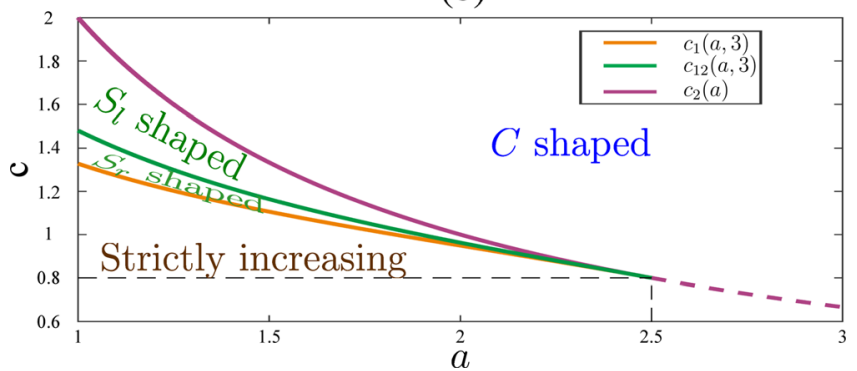

FIG. 4. Classification of endemic state curve $\tilde{S}$ on the $(a, c)$ and $(b, c)$ planes with the remaining parameter being fixed. In both panels, the graph of the corresponding $\tilde{S}$ either strictly increases or $C$ shaped as $b \leq b_{c}(=a+1 / 2)$. Otherwise, its graph could be either of the four shapes: strictly increasing, $S_{r}$ shaped, $S_{l}$ shaped, and $C$ shaped. (a) Set $a=1$. Then, $b_{c}=1.5$. The corresponding curves $c_{1}(1, b), c_{12}(1, b)$, and $c_{2}(1)$ colored by purple, green, and orange, respectively, divide the $(b, c)$ plane into 4 parts: strictly increasing, $C$ shaped, $S_{l}$ shaped, and $S_{r}$ shaped. The parameters chosen in Figs. 2(a) and 2(b) correspond to the red (resp., blue) dots in Fig. 3(a). (b) Set $b=3$. Then, for $a<2.5$ (resp., $a \geq 2.5$ ), we have that $b(=3)>b_{c}$ (resp., $b(=3) \leq b_{c}$ ). The $(a, c)$ plane is divided into 4 (resp., 2) parts whenever $a<2.5$ (resp., $a \geq 2.5$ ).

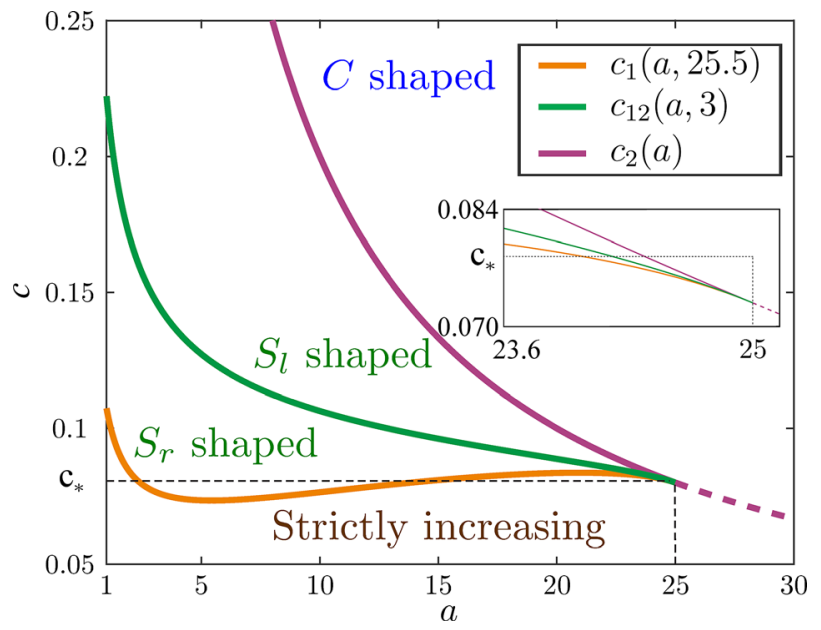

FIG. 5. Classification of $\tilde{S}$ on the $(a, c)$ plane with $b=25.5$ and $c<1$, which corresponds to the coinfection model of a mixed type. The graphs of $c_{1}(a, 25.5), c_{12}(a, 25.5)$, and $c_{2}(a)$ are colored by orange, green, and purple, respectively. For $c=0.082=: c_{*}$, as $\alpha$ gradually increases from 1 to 25 , the shape of $\tilde{S}$ changes from strictly increasing to $S_{r}$ shaped to strictly increasing to $S_{r}$ shaped to $S_{l}$ shaped to $C$ shaped. The zoom in version of the curves near $(a, c)=\left(25, c_{*}\right)$ is displayed on the middle right of the panel.

hysteresis of $S_{r}$ or $S_{l}$ type occurs. In summary, we see that the nonuniformity of the model is what drives the occurrence of the hysteresis.

The assumption in Theorem 2.2 is that $a, b \geq 1$. However, to apply the results in Theorem 2.2, $c$ is not required to be not smaller than one. So, we shall also explore the dynamics of (1) under a particular mixed type, i.e., $a$, $b \geq 1$ and $c \leq 1$. We see, via Figs. 5 and 6, that three types of hysteresis still exist, provided that the model is extremely nonuniform. For instance, with $(b, c)=(25.5,0.082)$, by increasing the nonuniformity measurement in $a$, we see that the model goes through the following phase transitions:

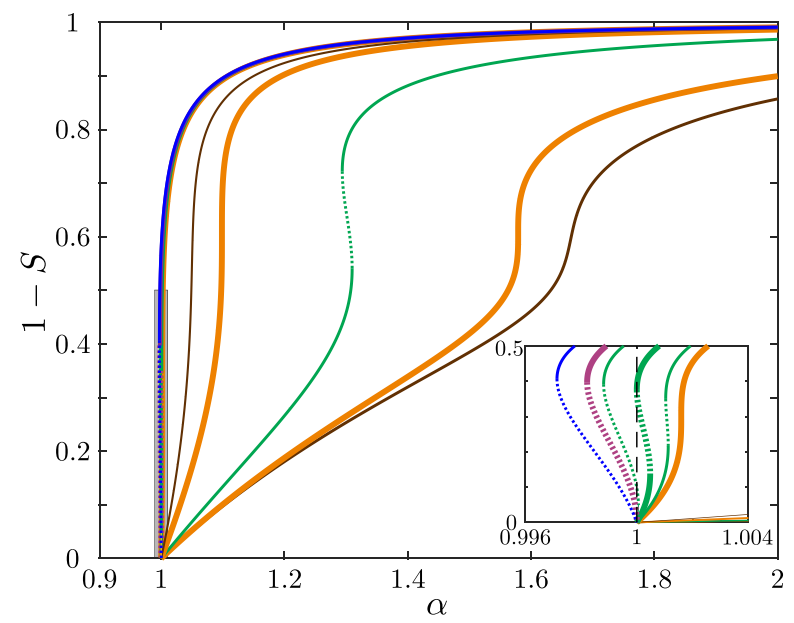

FIG. 6. Graphs of $\tilde{S}$ on the $(\alpha, 1-S)$ plane with fixed $b=25.5\left(=: b_{*}\right)$, $c=0.082\left(=: c_{*}\right)$ and varying $a>0$ discretely from $1.5<a_{1}<7<a_{2}<20$ $<a_{3}<24.1<a_{4}<24.33<a_{5}<24.5$. Here, $a_{1} \approx 2.1643, a_{2} \approx 16.4997$, and $a_{3} \approx 24.0397$ are the three positive roots of $c_{1}\left(a, b_{*}\right)=c_{*}$ (Fig. 5) and $a_{4} \approx 24.2080$ and $a_{5} \approx 24.3902$ are the roots of $c_{12}\left(a_{4}, b_{*}\right)=c_{*}$ and $c_{2}\left(a_{5}\right)=c_{*}$, respectively. The eleven curves in the panel from the right to the left correspond to various $a$ values as given above, respectively. The zoom in version of the curves near $\alpha=1$ is displayed on the lower right part of the panel. As $\alpha$ increases from 1 to 25 discretely, the shape of $\tilde{S}$ matches perfectly as mentioned in Fig. 5. 
continuous phase transition to hysteresis of $S_{r}$ type to continuous phase transition to hysteresis of $S_{r}$ type to hysteresis of $S_{l}$ type to hysteresis of $C$ type. We end this section by giving the following observations:

(A) If the devastation caused by the diseases is measured by how large the size of infected population jumps in the outbreak and by how much effort needed to eradicate the diseases, then hysteresis of $C$ (resp., $S_{r}$ ) type is the most (resp., least) devastating.

(B) The most interesting case, where $b>b_{c}$, exhibits four different regimes in the parameter space [Theorem 2.1, Figs. 4(a) and 4(b)]. Those behaviours can be understood by taking into account the microscopic features of the dynamics. In this situation, the cooperation mainly comes through state $\mathrm{AB}$, which is considerably more virulent (Table I). First, in the case that the value of $c$ is small, there are not enough $\mathrm{AB}$ states at the transition point in which the disease breaks out, and so, the transition is continuous. Moreover, enough $\mathrm{AB}$ states appear only when the system is already in a state with most of the nodes infected. This makes the effect on cooperation small, and hence, we have a continuous branch. If the value of $c$ is further increased, the appearance of more $\mathrm{AB}$ states is stimulated. Eventually, in the case that enough $\mathrm{AB}$ states appear after a threshold $\alpha_{o}$, which is greater than the point for the outbreak of diseases, is reached, cooperation for doubly infected $\mathrm{AB}$ infecting other states starts to be effective, and then, an abrupt transition emerges. Finally, if we keep on increasing $c$, the cooperation for secondary infection dominates the dynamics and the model matches the results reported in Ref. 26.

(C) In the case that the relative infection factor $a$ for the susceptible class is relatively high than that of $b$ for the singly infected class, or equivalently, $b \leq a+1 / 2$, there exist only two different regimes in the parameter space. The empirical reason for the disappearance of $S_{r}$ and $S_{l}$ shapes is due to the fact that a higher $a$ coupled with a larger $c$ would simulate enough states $\mathrm{AB}$ at the transition point where the disease breaks out. Consequently, an abrupt transmission emerges.

\section{WELL-MIXED SYSTEM: HETEROGENEOUS CASE}

In this section, we incorporate the heterogeneity in two diseases, i.e., different infection rates for the two diseases while keeping the same recovering rates. For simplicity, we assume that those infection rates for both diseases satisfy the following $\alpha_{B} / \alpha_{A}=\beta_{B} / \beta_{A} \equiv d$. Here, $d$ is a nonnegative real number. For $d>1$ (resp., $0<d<1$ ), we increase (decrease) the infection rate of disease $\mathrm{B}$ by a factor $d$. The equations defining the heterogeneous system are

$$
\begin{gathered}
\dot{S}(t)=-\alpha_{A} S\left(I_{A}+a I_{A B}\right)-d \alpha_{A} S\left(I_{B}+a I_{A B}\right)+I_{A}+I_{B}, \\
\dot{I}_{A}(t)=\alpha_{A} S\left(I_{A}+a I_{A B}\right)-d \beta_{A} I_{A}\left(I_{B}+b I_{A B}\right)+I_{A B}-I_{A}, \\
\dot{I}_{B}(t)=d \alpha_{A} S\left(I_{B}+a I_{A B}\right)-\beta_{A} I_{B}\left(I_{A}+b I_{A B}\right)+I_{A B}-I_{B}, \\
\dot{I}_{A B}(t)=d \beta_{A} I_{A}\left(I_{B}+b I_{A B}\right)+\beta_{A} I_{B}\left(I_{A}+b I_{A B}\right)-2 I_{A B} .
\end{gathered}
$$

To understand the dynamics of system (10), we first need to find an equation satisfied by $S$. Here, $E=\left(S, I_{A}\right.$, $\left.I_{B}, I_{A B}\right)$ is a fixed point of (10). In the homogeneous case, such an equation, given in (3), is a polynomial of degree 3 in $\alpha$ and $S$. For the heterogeneous case, such an equation is a polynomial equation of degree 5 in $\alpha_{A}$ and $S$, requiring more than a page to write it down (see supplementary material). This, in turn, makes the theoretical study of the dynamics of system (10) a daunting task. Hence, all the conclusions in this section are drawn from simulations and empirical evidences.

Our aim in this section is to understand how the measurement $d$ of heterogeneity in two diseases affects the dynamics of this model. To this end, we set $(a, b, c)=(1,2$, 2.03 ) and then numerically solve the $\alpha_{A}$ in terms of $S$. We then graph the resulting pair $\left(S, \alpha_{A}(S)\right)$ in the $\left(\alpha_{A}, 1-S\right)$ plane for various choices of $d$. Such a graph is denoted by $\Gamma_{d}$, where $d>0$ [Figs. 7(a) and 7(b)]. Denote by $\ell_{d}$ : $1-S=1-\frac{1}{d \alpha_{A}}$, where $\alpha_{A} \geq \frac{1}{d}$. For any $d>0, \ell_{1}$ corresponds to the set of all feasible trivial epidemic states $E_{A}$ $=\left(\frac{1}{\alpha_{A}}, 1-\frac{1}{\alpha_{A}}, 0,0\right)$, while $\ell_{d}$ is the set of all feasible epidemic states $E_{B}=\left(\frac{1}{d \alpha_{A}}, 0,1-\frac{1}{d \alpha_{A}}, 0\right)$. Note that the graph of $\ell_{1}$ lies above (resp., below) $\ell_{d}$ whenever $0<d<1$ (resp., $d>1$ ). Hence, it is reasonable to expect that the portion of $\Gamma_{d}$ lying on or above $\ell_{1}$ (resp., $\ell_{d}$ ) consists of feasible epidemic states whenever $0<d<1$ (resp., $d>1$ ). On the other hand, for $0<d<1$, the portion of $\Gamma_{d}$ lying below $\ell_{1}$ consists of infeasible epidemic states. Specifically, the states of $I_{B}$ associated with that portion of $\Gamma_{d}$ will become negative. Similarly, if $d>1$, then the portion of $\Gamma_{d}$ lying below $\ell_{d}$ has the state of $I_{A}$ to be negative. From here on, $\Gamma_{d}$ [Figs. 7(a) and $7(\mathrm{~b})]$ shall be referred to as its feasible part. In fact, $\Gamma_{d}$ is made up of the nontrivial epidemic states except at the interception point $H$ of $\Gamma_{d} \cap \ell_{d}$, where, in case that $0<d<1, \ell_{d}$ is to be interpreted as $\ell_{1}$. The $\alpha$-coordinate of $H$ is to be denoted by $\alpha_{d}$. For each $d>0$, the threshold for a discontinuous outbreak transition is denoted by $\alpha_{d, o}$.

In view of the shape of $\Gamma_{d} \cup \ell_{d}$ [Figs. 7(a) and 7(b)], we conjecture that the following hold true. (i) For $0<d<1$, the portion, denoted by $\ell_{d, A}$, of $\ell_{1}$ with $1 \leq \alpha_{A} \leq \alpha_{d}$ (resp., $\alpha_{A}>\alpha_{d}$ ) is stable (resp., unstable). Similarly, for $d>1$, the portion, denoted by $\ell_{d, B}$, of $\ell_{d}$ with $\frac{1}{d} \leq \alpha_{A} \leq \alpha_{d}$ (resp., $\alpha_{A}>\alpha_{d}$ ) is stable (resp., unstable). (ii) Define $d_{-k}=\frac{1}{d_{k}}$. For $d_{8} \approx 0.602<d<1$ (resp., $d_{-8}>d>1$ ), the corresponding epidemic states consist of two (solid) stable branches connected by a dotted unstable branch. The upper stable branch is only made up of nontrivial epidemic states. However, what the lower stable branch is made up of depends on $d$. In particular, for $1>d \geq d_{4} \approx 0.624$ and $1<d \leq d_{-4}$ $\approx 1.647$, the corresponding lower stable branch consists of disease free states $\left(0<\alpha_{A} \leq 1\right.$ and $\left.0<\alpha_{A} \leq \frac{1}{d}\right)$ and trivial epidemic states $\left(1<\alpha_{A} \leq \alpha_{d}\right.$ and $\left.\frac{1}{d}<\alpha_{A} \leq \alpha_{d}\right)$. In the above ranges of $d$, we have that $\alpha_{d, o}=\alpha_{d}$. For $d_{8}<d \leq d_{4}$ and $d_{-8}>d \geq d_{-4}$, its lower stable branch is made up of disease free states $\left(0<\alpha_{A} \leq 1\right.$ and $\left.0<\alpha_{A} \leq \frac{1}{d}\right)$, trivial epidemic states $\left(1<\alpha_{A} \leq \alpha_{d}\right.$ and $\left.\frac{1}{d}<\alpha_{A} \leq \alpha_{d}\right)$, and nontrivial epidemic states $\left(\alpha_{d}<\alpha_{A} \leq \alpha_{d, o}\right)$. (iii) For $0<d \leq d_{8}$ and 
(a)

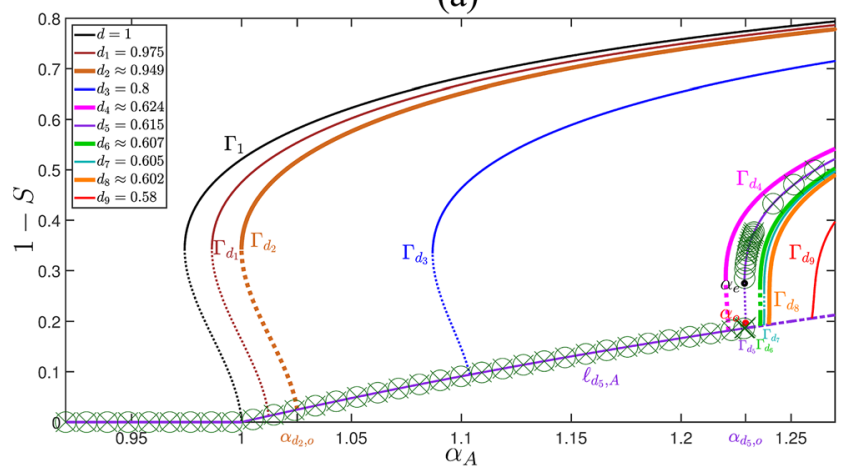

(b)

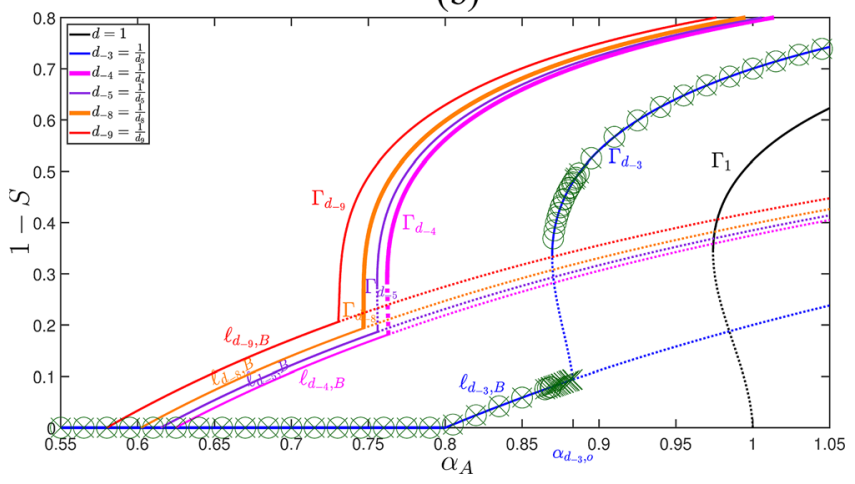

FIG. 7. Let $(a, b, c)=(1,2,2.03)$. Graphs of the fixed point curve corresponding to a heterogeneous model with the assumption that $\alpha_{B} / \alpha_{A}=\beta_{B} / \beta_{A} \equiv d$. Each curve consists of two stable branches, connected by a (dotted) unstable branch. For $d=d_{5}$ and $d_{-3}$, we run the numerical simulation of the corresponding heterogeneous model with two initial states $\left(I_{A}(0), I_{B}(0), I_{A B}(0)\right)=(0.005,0.005,0),(0.4,0.4,0.1)$. The resulting eventual states, stopped at $t=10^{4}$, are denoted by the circle and crosses, which match quite well with our predicted branches. (a) Let $0<d \leq 1$. The ten curves from left to right are the fixed point curve of the corresponding heterogeneous model $d$ varying from 1 to $d_{9}=0.58$. As $d$ gradually decreases to zero from one, $\Gamma_{d}$ changes from $C$ shaped to $S_{l}$ shaped to $S_{r}$ shaped to increasing. $d_{4}, d_{6}$, and $d_{8}$ correspond to the values of $d$ when such changes occur. The trivial epidemic curve $\ell_{1}: 1-S=1-\frac{1}{\alpha_{A}}$ corresponding to the trivial epidemic equilibrium $\left(S, I_{A}, I_{B}, I_{A B}\right)=\left(\frac{1}{\alpha_{A}}, 1-\frac{1}{\alpha_{A}}, 0,0\right)$ is independent of $d$. The threshold $\alpha_{d, o}$ for discontinuous outbreak transition increases as $d$ decreases from one. (b) Let $d>1$ and $d_{-k}=\frac{1}{d_{k}}$. For $d>1$, the trivial epidemic curves $\ell_{d}, 1-S=1-\frac{1}{d \alpha_{A}}$, corresponding to the trivial epidemic equilibrium $\left(S, I_{A}, I_{B}, I_{A B}\right)=\left(\frac{1}{d \alpha_{A}}, 0,1-\frac{1}{d \alpha_{A}}, 0\right)$, depend on $d$. The six fixed point curves (from left to right) correspond to various $d$ values as indicated in Fig. 7(b). The threshold $\alpha_{d, o}$ for discontinuous outbreak transition $\alpha_{d, o}$ decreases as $d$ increases from one. For $1<d<d_{-4}, \Gamma_{d}$ is a $C$-shaped. For $d_{-4}<d<d_{-8}, \Gamma_{d}$ is a $S$-shaped. For $d>d_{-8}$, the corresponding fixed point curve increases.

$d \geq d_{-8}$, the unstable branch disappears and two stable branches merge, which make system (10) noninteracting. In summary, the differences in dynamics between homogeneous and heterogeneous models stem from the fact that trivial epidemic states of the former remain unstable for all $\alpha \geq 1$, while the trivial epidemic states of the later growing initially from disease free states must be stable.

To verify the stability of each branch, we set additionally $d=d_{5}=0.615$ or $d=d_{-3}=\frac{5}{4}$ and start out with two sets of initial conditions: $\left(I_{A}(0), I_{B}(0), I_{A B}(0)\right)=(0.005$, $0.005,0)$ and $\left(I_{A}(0), I_{B}(0), I_{A B}(0)\right)=(0.4,0.4,0.1)$, which correspond to the early stage of epidemic and the outbreak of the diseases, respectively. We then integrate system (10) and report the converging values for the initial values. The corresponding eventual states, stopped at $t=10^{4}$, are marked by circles and crosses, respectively, which match quite well with predicted stable branches [Fig. 7(a)]. In any event, we see, via Figs. 7(a) and 7(b), that the threshold $\alpha_{d, o}$ for a discontinuous outbreak transition will increase (resp., decrease) as $d$ gradually decreases (resp., increases) from one. The empirical explanations of the above phenomena are to be explained in the next paragraph.

For a homogeneous coinfection model, i.e., $d=1$ and $(a, b, c)=(1,2,2.03)$, both diseases are at the cusp of having stable infected population when $\alpha_{A}=\alpha_{B}=1$, which is also when the cooperation of two diseases is at its full strength. Hence, as $\alpha$ races pass one, the discontinuous jump of infected population, which forms a hysteresis loop of type $C$, can be seen. However, for $d<1$, due to the heterogeneity in two diseases, disease $\mathrm{A}$ is ready to produce stable infected population at $\alpha_{A}=1$, while disease $\mathrm{B}$ is not. Hence, to have a sudden jump in the infected population, disease $\mathrm{A}$ has to produce more stable infected population so that the infected population for disease B can be stabilized due to their cooperation. Once both diseases can produce stabilized infected population, a discontinuous jump in the total infected population can then be observed. As the imbalance of the transmission rates between two diseases grows, i.e., $d$ becomes smaller, disease A needs to produce even a greater number of infected population in order to generate stable infected population for disease B. Specifically, if $1 \geq d>d_{4}$, then the discontinuous jump occurs at $\alpha_{d, o}=\alpha_{d}$, see, e.g., $\Gamma_{d 1}, \Gamma_{d 2}, \Gamma_{d 3}$ in Fig. 7(a). For $d_{8}<d<d_{4}$, meaning that the infection rate for disease $B$ is further weaken, the discontinuous jump has to further wait until the nontrivial epidemic states can also be stabilized. Similar empirical interpretations can then be given for $d>1$. In fact, if we switch the labels of diseases A and B in Eq. (10), then the corresponding ratios of $\alpha_{B} / \alpha_{A}$ and $\beta_{B} / \beta_{A}$ become $1 / d$. That is to say, system (10) with $d>1$ and $0<d<1$ is mathematically equivalent.

One final remark about the heterogeneity in two diseases is that the hysteresis loop of $C$ shaped completely disappears whenever $d \neq 1$. Such an observation is based on the conjecture (i) mentioned earlier and in Figs. 7(a) and 7(b).

\section{CONCLUSION}

In this paper, we propose a fully cooperative coinfection model, which is investigated through the well-mixed approach. We prove analytically that the model exhibits three types of hysteresis, provided that the model is relatively nonuniform in its parameters. In particular, we prove that discontinuous outbreak transitions from the disease free or low prevalence state to the high prevalence state can be separately observed as a disease transmission rate crosses a threshold $\alpha_{o}$ from the below when the epidemic is still in the early stages and that discontinuous eradications from the high prevalence state to the low prevalence or disease free state are also separately seen as the transition rate reaches a threshold to $\alpha_{e}\left(<\alpha_{o}\right)$ from the above when the outbreak 
occurs. Such phenomena are sharply in contrast to a single disease model which only exhibits a continuous transition. Moreover, the results also highlight the importance of preventing the outbreak of disease since it would then take greater effort to eradicate it.

The effect of heterogeneity in two diseases observed in Sec. III is based on simulations. It is, therefore, valuable to analytically verify the results mentioned there. It is of great interest to study the effect of vaccination and awareness on such a fully cooperative coinfection model. The network based approach for coinfection models with or without delay is also worthwhile to pursue.

\section{SUPPLEMENTARY MATERIAL}

See supplementary material for equations to solve fixed points for the heterogeneous case considered in Sec. III.

\section{ACKNOWLEDGMENTS}

J.P.R. acknowledges support from the Ministry of Science and Technology (Taiwan) and the FPU program of MECD (Spain), received funding from SPASIMM [FIS2016-80067-P (AEI/FEDER, UE)], and thanks Víctor M. Eguíluz and Fakhteh Ghanbarnejad for comments and discussion. Y.H.L. and J.J. acknowledge support from the Ministry of Science and Technology of Taiwan under Grant No. Most 105-2115-M-009-002-MY2.

\section{APPENDIX: PROOFS OF SOME CLAIMS IN SEC. II}

In the Appendix, we provide the rigorous proofs for some claims in Sec. II. We begin with proving some properties of fixed points of (1).

Proposition A.1. Let $E^{*}=\left(S^{*}, I_{A}^{*}, I_{B}^{*}, I_{A B}^{*}\right)$ be a fixed point of (1). Then, if $E^{*}$ is a nontrivial endemic fixed point, i.e., $\left(I_{A}^{*}\right)\left(I_{B}^{*}\right)\left(I_{A B}^{*}\right) \neq 0$, then $I_{A}^{*}=I_{B}^{*}$. Otherwise, $E^{*}=(1,0$, $0,0),\left(\frac{1}{\alpha}, 1-\frac{1}{\alpha}, 0,0\right)$ or $\left(\frac{1}{\alpha}, 0,1-\frac{1}{\alpha}, 0\right)$.

Proof. To prove this proposition, we first show some basic properties of solutions of (1). Let $E(t)=\left(S(t), I_{A}(t)\right.$, $\left.I_{B}(t), I_{A B}(t)\right)$ be an arbitrary solution of (1). Then, either one of the following cases occurs: (A) $S(t) \geq \frac{1}{\alpha}$ for any $t \geq 0$ or (B) there exists $t_{0} \geq 0$ such that $S\left(t_{0}\right)<\frac{1}{\alpha}$. If case (A) holds, we compute that

$$
\begin{aligned}
\dot{S}(t) & \leq-\left(I_{A}+a I_{A B}\right)-\left(I_{B}+a I_{A B}\right)+I_{A}+I_{B} \\
& =-2 a I_{A B} \leq 0
\end{aligned}
$$

for any $t \geq 0$. Applying Barbalet's Lemma, we see that $\lim _{t \rightarrow \infty} \dot{S}(t)=0$, and hence, $\lim _{t \rightarrow \infty} I_{A B}(t)=0$. If, on the other hand, case (B) occurs, then $S(t) \leq \frac{1}{\alpha}$ for any $t \geq t_{0}$. Indeed, in contrast, there exists $t_{1} \geq t_{0}$ such that $S\left(t_{1}\right) \geq \frac{1}{\alpha}$ and $S^{\prime}\left(t_{1}\right)>0$, a contraction to (A1). Then, defining a Lyapunov function $V(t)=\frac{1}{2}\left(I_{A}(t)-I_{B}(t)\right)^{2}$, we have that

$$
\begin{aligned}
\dot{V}(t) & =\left(\alpha S-1-\beta b I_{A B}(t)-1\right)\left(I_{A}(t)-I_{B}(t)\right)^{2} \\
& \leq-\beta b I_{A B}(t)\left(I_{A}(t)-I_{B}(t)\right)^{2} \leq 0
\end{aligned}
$$

for any $t \geq t_{0}$. Similarly, applying Barbalet's Lemma, we get that $\lim _{t \rightarrow \infty} I_{A B}(t)=0$ or $\lim _{t \rightarrow \infty}\left(I_{A}(t)-I_{B}(t)\right)=0$.

We are now in a position to prove the assertion of this proposition. Let $E(t)$ be the solution of (1) with its initial state being the fixed point $E^{*}=\left(S^{*}, I_{A}^{*}, I_{B}^{*}, I_{A B}^{*}\right)$. Then, clearly, $E(t) \equiv E^{*}$ for all $t \geq 0$. Moreover, if $S^{*} \geq \frac{1}{\alpha}$, then $S(t) \equiv S^{*}$ $\geq \frac{1}{\alpha}$, and hence, $I_{A B}^{*}=\lim _{t \rightarrow \infty} I_{A B}(t)=0$, as claimed in case (A). On the other hand, if $S^{*}<\frac{1}{\alpha}$, then $S(t) \equiv S^{*}<\frac{1}{\alpha}$, and hence, $I_{A B}^{*}=0$ or $I_{A}^{*}=I_{B}^{*}$, as claimed in case (B). In summary, if $E^{*}$ is a nontrivial endemic fixed point, then $I_{A}^{*}=I_{B}^{*}$; otherwise, $I_{A B}^{*}=0$, and it can then be proved easily, via (1), that at least one of the conditions $I_{A}^{*}=0$ and $I_{B}^{*}=0$ holds, which completes the proof of the proposition.

We next show that the solutions $(S, \alpha)$ of $f(S, \alpha)=0$ with $0<S<1$ implicitly define a function $\bar{\alpha}(s)$ from $(0,1 / S]$ such that $f(S, \bar{\alpha}(S))=0$.

Proposition A.2. Fix $a, b \geq 1$ and $c>0$. Then, for any $0<S \leq 1$, there exists a unique $\bar{\alpha}(=\bar{\alpha}(S))$ on $(0,1 / S]$ such that $f(S, \bar{\alpha}(S))=0$.

Proof. Fix $a, b \geq 1$ and $c>0$. We first consider the case $0<S<1$. Writing $f(S, \alpha)$ as $g_{S}(\alpha)$, we have that $g_{S}(0)$ $=-1$ and $g_{S}(1 / S)=a^{2} c(1-S) / S>0$ for $0<S<1$. Hence, there must exist $\bar{\alpha}$ on $(0,1 / S)$ such that $g_{S}(\bar{\alpha})=0$. Suppose that there exist two roots $\alpha_{1}$ and $\alpha_{2}$ of $g_{S}(\alpha)$ on $(0,1 / S)$. Then, since $g_{S}(0)=-1<0$ and $g_{S}^{\prime}(0)=-2(a-1)$ $S \leq 0$, there exists a critical point on $\left[0, \alpha_{1}\right)$. Moreover, another critical point should exist on $\left(\alpha_{1}, \alpha_{2}\right)$. Noting $g_{S}(1 / S)>0$ for $0<S<1$, we also conclude that a third critical point exists on $\left(\alpha_{2}, 1 / S\right)$, a contradiction to the fact that $g_{S}(\alpha)$ is a cubic polynomial. Suppose there exist three roots on $(0,1 / S)$. Upon using the fact that $g_{S}(0)<0$, we conclude that $g_{S}(\alpha)$ must be increasing on $(-\infty, 0)$, a contradiction to the fact that $g_{S}^{\prime}(0) \leq 0$. Therefore, $g_{S}(\alpha)$ has a unique root on $(0,1 / S)$ for $0<S<1$. For $S=1$, the assertion of the proposition can be verified directly.

We next show that the graphs of $\bar{\alpha}(S)$ and $\tilde{\alpha}(T)$ defined in (5) have the same shape on the interval of $(0,1]$ in the following sense. Note that the transformation given in (4) defines a bijective mapping between $(S, \bar{\alpha}(S))$ and $(T, \tilde{\alpha}(T))$.

Proposition A.3. For any $0<t<1$, we have that

$$
\operatorname{sgn}\left(\left[\frac{d}{d T} \tilde{\alpha}(T)\right]_{T=t}\right)=\operatorname{sgn}\left(\left[\frac{d}{d S} \bar{\alpha}(S)\right]_{S=\frac{t}{\bar{\alpha}(t)}}\right) .
$$

Here, function $\operatorname{sgn}(x)$ takes the sign of $x$.

Proof. By the chain rule, we have that for any $0<t<1$

$$
\left[\frac{d}{d S} \bar{\alpha}(S)\right]_{S=\frac{t}{\bar{\alpha}(t)}}=\left[\frac{\frac{d}{d T} \tilde{\alpha}(T)}{\frac{d}{d T}\left[\frac{T}{\tilde{\alpha}(T)}\right]}\right]_{T=t} .
$$

To complete the proof, it suffices to show that $\frac{d}{d T}\left[\frac{T}{\tilde{\alpha}(T)}\right]>0$ for all $0<T<1$. Indeed, some direct computation yield that

$$
\frac{d}{d T}\left[\frac{T}{\tilde{\alpha}(T)}\right]=\frac{N_{1}(T, a, b)}{a c T[\tilde{\alpha}(T)]^{2}[(a-b) T+b]^{2}},
$$


where

$$
\begin{aligned}
N_{1}(T, a, b)= & {\left[2(2-T) T^{2}\right] a^{2} } \\
& +\{T(1-T)[2 b(1-T)+(3-T)]\} a \\
& +b(2-T)(T-1)^{2}>0 .
\end{aligned}
$$

We have just completed the proof of the proposition.

To see the shape of the graph of $\tilde{\alpha}(T)$, we first compute the derivatives of $\tilde{\alpha}(T)$ at its two end points, 0 and 1 .

Proposition A.4. The following assertions hold:

(i) $\lim _{T \rightarrow 0^{+}} \tilde{\alpha}(T)=\infty$ and $\tilde{\alpha}(1)=1$.

(ii) $\lim _{T \rightarrow 0^{+}} \tilde{\alpha}^{\prime}(T)=-\infty$ and $\tilde{\alpha}^{\prime}(1)=1-\frac{2}{a c}$. In particular, if $c=\frac{2}{a}$, then $\tilde{\alpha}^{\prime}(1)=0$.

(iii) $\lim _{T \rightarrow 0^{+}} \tilde{\alpha}^{\prime \prime}(T)=\infty$ and $\tilde{\alpha}^{\prime \prime}(1)=-\frac{4}{a^{2} c}[b-a-1 / 2]$. Consequently, if $b \leq b_{c}$, then $\tilde{\alpha}^{\prime \prime}(T)>0$ on $(0,1)$.

(iv) $\quad \tilde{\alpha}^{\prime \prime \prime}(T)<0$ for all $0<T \leq 1$ and $a \geq 1$.

Proof. Some direct computations yield that

$$
\begin{aligned}
\tilde{\alpha}^{\prime}(T) & =\frac{M_{1}(T, a, b, c)}{a c T^{2}[(a-b) T+b]^{2}}, \\
\tilde{\alpha}^{\prime \prime}(T) & =2 \frac{M_{2}(T, a, b)}{a c T^{3}[(a-b) T+b]^{3}}, \\
\tilde{\alpha}^{\prime \prime \prime}(T) & =6 \frac{M_{3}(T, a, b)}{a c T^{4}[(a-b) T+b]^{4}},
\end{aligned}
$$

where

$$
\begin{aligned}
M_{1}(T, a, b, c)= & {\left[a(a-b)^{2} c\right] T^{4}+[2 a b(a-b) c] T^{3} } \\
& +\left[a b^{2} c-2 a^{2}+2 a-b\right] T^{2} \\
& +2(b-a) T-b, \\
M_{2}(T, a, b)= & {\left[(a-b)\left(2 a^{2}-2 a+b\right)\right] T^{3} } \\
& +3(a-b)^{2} T^{2}+3 b(a-b) T+b^{2}, \\
M_{3}(T, a, b)= & -\left[(a-b)^{2}\left(2 a^{2}-2 a+b\right)\right] T^{4} \\
& -4(a-b)^{3} T^{3}-6 b(a-b)^{2} T^{2} \\
& -4 b^{2}(a-b) T-b^{3} .
\end{aligned}
$$

Then, the assertions of parts (i)-(iii) in the proposition can be verified directly. To see (iv), we rewrite $M_{3}(T, a, b)$ as the polynomial form of $b$. Specifically, we have that

$$
\begin{aligned}
M_{3}(T, a, b) & =U_{3} b^{3}+U_{2} b^{2}+U_{1} b+U_{0} \\
& =b^{2}\left[U_{0} b^{-2}+U_{1} b^{-1}+U_{2}\right]+U_{3} b^{3},
\end{aligned}
$$

where $U_{3}=-(1-T)^{4}(<0), U_{2}=-2 a T\left[(a-2) T^{3}+6 T^{2}\right.$ $-6 T+2], U_{1}=a^{2} T^{2}\left[(4 a-5) T^{2}+12 T-6\right]$, and $U_{0}=-2 a^{3} T^{3}$ $[(a-1) T+2](<0)$. Hence, to conclude the assertion (iv) in the proposition, we only need to show that $U_{1}^{2}-4 U_{0} U_{2}<0$ for all $0<T \leq 1$ and $a \geq 1$. Indeed, we compute that $U_{1}^{2}$ $-4 U_{0} U_{2}=a^{4} T^{4}\left[(8 a-7)(1-T)^{4}-12(1-T)^{3}-6(1-T)^{2}\right.$ $-4(1-T)+(1-8 a)] \leq a^{4} T^{4}[(8 a-7)+(1-8 a)]=-6 a^{4} T^{4}$ $<0$. The proof is now complete.
Proposition A.5. For any $a \geq 1$ and $b \leq b_{c}(:=a+1 / 2)$, the following two assertions hold:

(i) If $c \leq c_{2}$, then $\tilde{\alpha}(T)$ strictly decreases on $(0,1]$.

(ii) If $c>c_{2}$, then $\tilde{\alpha}(T)$ has a unique minimum on $(0,1)$. In particular, the graph of $\tilde{\alpha}(T)$ is $C$ shaped.

Here, $c_{2}=\frac{2}{a}$ is defined as in (6).

Proof. Since $b \leq b_{c}$, we have that $\tilde{\alpha}^{\prime \prime}(1) \geq 0$ by Proposition A.4(iii). Consequently, $\tilde{\alpha}^{\prime \prime}(T)>0$ for all 0 $<T<1$ by Proposition A.4(iv), which implies that $\tilde{\alpha}(T)$ is concave up on $(0,1]$. In addition, $\tilde{\alpha}(T)$ strictly decreases on $(0,1]$ if $\tilde{\alpha}^{\prime}(1) \leq 0$, while first decreasing and then increasing on $(0,1]$ if $\tilde{\alpha}^{\prime}(1)>0$. The above cases correspond to $c \leq c_{2}$ or $c>c_{2}$, respectively, by Proposition A.4(ii). Hence, the proof is complete.

For $a \geq 1$ and $b>b_{c}$, the graph of $\tilde{\alpha}(T)$ is more complicated since the concavity of $\tilde{\alpha}(T)$ changes from the upward to the downward on $(0,1)$ by Proposition A.4(iii) and (iv). More precisely, $\tilde{\alpha}(T)$ has an inflection point $\gamma$ on $(0,1)$ such that

$$
\tilde{\alpha}^{\prime \prime}(T)\left\{\begin{aligned}
>0 & \text { if } T \in(0, \gamma), \\
=0 & \text { if } T=\gamma, \\
<0 & \text { if } T \in(\gamma, 1] .
\end{aligned}\right.
$$

To classify its shape, we first find $c$ so that the corresponding $\tilde{\alpha}(T)$ has some particular properties.

Proposition A.6. For any $a \geq 1$ and $b>b_{c}(:=a+1 / 2)$, define $\gamma$ as in (A4). Then, the following three assertions hold:

(i) There exists a unique positive $c_{1}$, defined as in (7), such that

$$
\tilde{\alpha}^{\prime}(\gamma)=\tilde{\alpha}^{\prime \prime}(\gamma)=0 .
$$

In particular, $c_{1}$ is chosen as the property stated in Theorem 2.1(II) is satisfied.

(ii) There exists a unique positive $c_{12}$, defined as in (8), such that the equation

$$
\tilde{\alpha}(T)=1(=\tilde{\alpha}(1))
$$

In particular, if $c_{12}$ possesses the property as stated in Theorem 2.1(II), then (A6) has a unique solution on $(0,1)$ when $c=c_{12}$. Moreover, (A6) has no solution on $(0,1)$ when $c<c_{12}$.

(iii) It holds that $c_{1}<c_{12}<c_{2}$. Here, $c_{2}=\frac{2}{a}$ is defined as in (6).

Proof. Fix $a \geq 1$ and $b>b_{c}(:=a+1 / 2)$. We first show Proposition A.6(i). Since $\gamma$ is the inflection point of $\tilde{\alpha}$, it holds that $\tilde{\alpha}^{\prime \prime}(\gamma)=0$. We have, via (A3), that $M_{2}(\gamma, a, b)$ $=0$. Moreover, since function $M_{2}$ is independent of $c$, we have that $\gamma$ is also independent of $c$. On the other hand, note that

$$
\operatorname{sgn}\left(\tilde{\alpha}^{\prime}(T)\right)=\operatorname{sgn}\left(M_{1}(T, a, b, c)\right)
$$

by (A3), and $M_{1}(T, a, b, c)$ can be rewritten as 


$$
\begin{aligned}
M_{1}(T, a, b, c)= & {\left[a T^{2}(a T-b T+b)^{2}\right] c } \\
& -\left[\left(2 a^{2}-2 a+b\right) T^{2}+2(b-a) T+b\right] \\
= & : \alpha_{1}(T) c-\alpha_{0}(T) .
\end{aligned}
$$

Clearly, $\alpha_{1}(T)$ and $\alpha_{0}(T)$ are positive for all $0<T<1$. By choosing $c_{1}=\frac{\alpha_{0}(\gamma)}{\alpha_{1}(\gamma)}$, we have that

$$
\tilde{\alpha}^{\prime}(\gamma) \begin{cases}<0 & \text { if } c<c_{1}, \\ =0 & \text { if } c=c_{1}, \\ >0 & \text { if } c>c_{1} .\end{cases}
$$

In fact, we can obtain the explicit form of $c_{1}$, as given in (7) by using the Maple.

We next show Proposition A.6(ii). By solving $\tilde{\alpha}(T)=1$ directly, we have that $T=1$ or

$$
T=\frac{(a b c-2 a+1) \pm \sqrt{L(a, b, c)}}{2 a(b-a) c}=: \hat{T},
$$

where

$L(a, b, c)=\left(a^{2} b^{2}\right) c^{2}+\left(-4 a^{2} b+4 a^{2}-2 a b\right) c+(2 a-1)^{2}$.

Hence, for equation $\tilde{\alpha}(T)=1$ to have exactly one solution on $\mathbb{R}$, we must have $L(a, b, c)=0$ or, equivalently

$$
c=\frac{(2 a b-2 a+b) \pm 2 \sqrt{a(2 b-1)(b-a)}}{a b^{2}}=: c_{ \pm} .
$$

We are then able to derive the following results.

(a) For $c=c_{-}$, we have that $\hat{T}=\frac{\left(a b c_{-}-2 a+1\right)}{2 a(b-a) c_{-}}<0$ by some tedious computation. It implies that $\tilde{\alpha}(T)$ is either greater or less than 1 on the entire interval $(0,1)$. In fact, $\tilde{\alpha}(T)>1$ on $(0,1)$ since $\lim _{T \rightarrow 0^{+}} \tilde{\alpha}(T)=\infty$ by Proposition A.4(i).

(b) For $c<c_{-}$, we have, via direct computation, that

$$
\frac{\partial}{\partial c} \tilde{\alpha}(T)=-\frac{(1-T)[2 a T+(1-T)]}{a c^{2} T[a T+b(1-T)]}<0
$$

whenever $0<T<1$. This implies that $[\tilde{\alpha}(T)]_{c}$ $>[\tilde{\alpha}(T)]_{c=c_{-}}>1$ for $c<c_{-}$.

(c) For $c \in\left(c_{-}, c_{+}\right)$, since $L(a, b, c)<0$, equation $\tilde{\alpha}(T)=1$ has no solution on $\mathbb{R}$ and hence $[\tilde{\alpha}(T)]_{c}>1$ on $(0,1)$.

(d) For $c=c_{+}$, we have that $\hat{T}=\frac{\left(a b c_{+}-2 a+1\right)}{2 a(b-a) c_{+}} \in(0,1)$ by some tedious computation. Moreover, since $[\tilde{\alpha}(T)]_{c}$ $>1$ on $(0,1)$ for $c<c_{+}$as claimed above, we have that $[\tilde{\alpha}(T)]_{c_{+}} \geq 1$ on $(0,1)$ and the equality holds if and only if $T=\hat{T}$.

Hence, the proof of Proposition A.6(ii) is complete by choosing $c_{12}=c_{+}$.

Finally, we show Proposition A.6(iii). As in the proof of part (ii), we have that $\tilde{\alpha}(T)>1$ on $(0,1)$ whenever $c<c_{12}$; while $\tilde{\alpha}(T) \geq 1$ on $(0,1)$, there exists $T_{*}$ on $(0,1)$ such that $\tilde{\alpha}\left(T_{*}\right)=1$ whenever $c=c_{12} ; \tilde{\alpha}(T)<1$ for some point on $(0,1)$ whenever $c>c_{12}$ by (A6) and (A9). Then, by (A4) and (A5), we have that, when $c=c_{1}, \tilde{\alpha}(T)$ strictly decreases on $(0,1)$, and hence, $\tilde{\alpha}(T)>\tilde{\alpha}(1)(=1)$ on $(0,1)$. So $c_{1}<c_{12}$. On the other hand, since $\tilde{\alpha}^{\prime}(1)=0$ by Proposition A.4(ii) and $\tilde{\alpha}^{\prime \prime}(1)<0$ by (A4), we have that $\tilde{\alpha}^{\prime}(T)<0$, and hence, $\tilde{\alpha}(T)<\tilde{\alpha}(1)(=1)$ whenever $T$ is sufficiently close to $1^{-}$. So, $c_{2}>c_{12}$.

We have just completed the proof of Proposition A.6.

Proposition A.7. For any $a \geq 1$ and $b>b_{c}(:=a$ $+1 / 2$ ), the following four assertions hold:

(i) If $c<c_{1}$, then $\tilde{\alpha}(T)$ strictly decreases.

(ii) If $c \in\left(c_{1}, c_{12}\right)$, then $\tilde{\alpha}(T)$ has a unique local minimum and a unique local maximum on $(0,1)$. The graph of such $\tilde{\alpha}(T)$ is to be called an $S$ shaped. Moreover, we have that $\tilde{\alpha}(T)>1$ for all $0<T<1$.

(iii) If $c \in\left(c_{12}, c_{2}\right)$, then the graph of $\tilde{\alpha}(T)$ is also $S$ shaped with $\tilde{\alpha}(T)<1$ for some $T$ on $(0,1)$.

(iv) If $c>c_{2}$, then $\tilde{\alpha}(T)$ has a unique critical point. The graph of such $\tilde{\alpha}(T)$ is to be termed a $C$ shaped.

Proof. We first prove Proposition A.7(i). By (A4) and (A5), we have that, when $c=c_{1}, \tilde{\alpha}^{\prime}(T) \leq 0$ on $(0,1)$. Then, by (A7), we have that $\tilde{\alpha}^{\prime}(T)<0$ on $(0,1)$ if $c<c_{1}$. So, the assertion of part (i) holds.

We next prove Proposition A.7(ii) and (iii). For $c \in\left(c_{1}, c_{2}\right)$, we have that $\tilde{\alpha}^{\prime}(\gamma)>0$ by (A8) and $\tilde{\alpha}^{\prime}(1)<0$ by Proposition A.4(ii). Then, by (A4), we conclude that $\tilde{\alpha}(T)$ has a unique local minimum and a unique local maximum on $(0,1)$. The remaining parts of the proof have been claimed in the proof of Proposition A.6(iii). So, the assertions of parts (ii) and (iii) hold.

Finally, we prove Proposition A.7(iv). In fact, the assertion holds by (A4) since $\tilde{\alpha}^{\prime}(\gamma)>0$ by (A8) and $\tilde{\alpha}^{\prime}(1)>0$ by Proposition A.4(ii).

We have just completed the proof of Proposition A.7.

The proof of the stability of fixed points of Eq. (1) is provided in the following:

Proof of Theorem 2.2. The proof of Theorem 2.2(i) is trivial, and hence, we omit it here.

We next consider the stability of $E^{*}=\left(\frac{1}{\alpha}, 1-\frac{1}{\alpha}, 0,0\right)$ (Similar arguments below can be applied to $E^{*}=\left(\frac{1}{\alpha}, 0\right.$, $\left.\left.1-\frac{1}{\alpha}, 0\right)\right)$. Note first that since the total population is conserved, system (1) can be reduced to a system of three unknowns, say $S(t), I_{A}(t)$, and $I_{B}(t)$. A direct computation yields that the linearized matrix at $E^{*}$ is of the form

$$
\boldsymbol{H}=\left[H_{i j}\right]_{1 \leq i, j \leq 3},
$$

where

$$
\begin{aligned}
& H_{11}=2 a-\alpha+1, \quad H_{12}=H_{13}=2 a, \\
& H_{21}=-2-a+\alpha+b c(\alpha-1), \\
& H_{22}=-1+b c(\alpha-1)-a \\
& H_{23}=H_{21}+1-\alpha-c \alpha+c, \\
& H_{31}=H_{32}=-1-a, \quad H_{33}=-1-a-c \alpha+c .
\end{aligned}
$$

Moreover, the characteristic polynomial of $\boldsymbol{H}$ is equal to

$$
\operatorname{det}(\boldsymbol{H}-\lambda \boldsymbol{I})=-(\lambda-(1-\alpha)) \operatorname{det}(\hat{\boldsymbol{H}}-\lambda \boldsymbol{I}),
$$


where

$$
\hat{\boldsymbol{H}}=\left[\begin{array}{cc}
-1+a+b c \alpha-b c & \hat{H}_{11}+c-c \alpha \\
-1-a & -1-a-c \alpha+c
\end{array}\right] .
$$

Since

$$
\operatorname{det}(\hat{\boldsymbol{H}})=-c(\alpha-1)[b c(\alpha-1)+2 a]<0,
$$

$\hat{\boldsymbol{H}}$ and hence $\boldsymbol{H}$ are Hurwitz matrices, which completes the proof of Theorem 2.2(ii).

Finally, we consider the stability of nontrivial endemic fixed point $E^{*}=\left(S^{*}, I_{A}^{*}, I_{B}^{*}, I_{A B}^{*}\right)$. We first compute that, via (2a) and by the fact that $I_{A}^{*}=I_{B}^{*}$ (Proposition A.1), the linearized matrix at $E^{*}$ is given as follows:

$$
\boldsymbol{M}=\left[M_{i j}\right]_{1 \leq i, j \leq 3},
$$

where

$$
\begin{aligned}
M_{11}= & -2 a \alpha+4 a \alpha S^{*}-2(1-2 a) \alpha I_{A}^{*}, \\
M_{12}= & M_{13}=1-(1-2 a) \alpha S^{*}, \\
M_{21}= & M_{31}=-(1-a \alpha)-2 a \alpha S^{*}+[(1-2 a)+b c] \alpha I_{A}^{*}, \\
M_{32}= & M_{23}=-1-a \alpha S^{*}-(1-b) c \alpha I_{A}^{*}, \\
M_{22}= & M_{33}=-(2+b c \alpha) \\
& +[(1-a)+b c] \alpha S^{*}-(1-3 b) c \alpha I_{A}^{*} .
\end{aligned}
$$

Moreover, the characteristic polynomial of $\boldsymbol{M}$ is equal to

$$
\operatorname{det}(\boldsymbol{M}-\lambda \boldsymbol{I})=(m-\lambda) \operatorname{det}(\hat{\boldsymbol{M}}-\lambda \boldsymbol{I})
$$

where

$$
\begin{aligned}
m & =\left(\alpha S^{*}-1\right)+b c \alpha\left(S^{*}+2 I_{A}^{*}-1\right) \\
& =\left(\alpha S^{*}-1\right)-b c \alpha I_{A B}^{*}<0,
\end{aligned}
$$

by (2b), and $\hat{\boldsymbol{M}}=\left[\hat{M}_{i j}\right]_{1 \leq i, j \leq 2}$ with

$$
\begin{aligned}
& \hat{M}_{11}=-2 a \alpha+4 a \alpha S^{*}-2(1-2 a) \alpha I_{A}^{*}, \\
& \hat{M}_{12}=1-(1-2 a) \alpha S^{*}, \\
& \hat{M}_{21}=-2(1-a \alpha)-4 a \alpha S^{*}+2[(1-2 a)+b c] \alpha I_{A}^{*}, \\
& \hat{M}_{22}=-(3+b c \alpha)+[(1-2 a)+b c] \alpha S^{*}-(2-4 b) c \alpha I_{A}^{*} .
\end{aligned}
$$

Hence, $E^{*}$ is locally stable, provided that the $\operatorname{trace} \operatorname{tr}(\hat{\boldsymbol{M}})$ and the determinant $\operatorname{det}(\hat{\boldsymbol{M}})$ of $\hat{\boldsymbol{M}}$ are negative and positive, respectively. Moreover, $E^{*}$ is unstable, $\operatorname{provided}$ that $\operatorname{det}(\hat{\boldsymbol{M}})$ $>0$. In view of the form of $\tilde{\alpha}^{\prime}(T)$, given as in (A2) and (A3), our next step toward completing the proof is to write $\operatorname{tr}(\hat{\boldsymbol{M}})$ and $\operatorname{det}(\hat{\boldsymbol{M}})$ in terms of $T^{*}\left(=: \alpha S^{*}\right), a, b$, and $c$ only. After lengthy and tedious calculations, we have, via (4), (5), and (2b), that

$$
\begin{aligned}
m_{1} \hat{M}_{11}= & \left(2 a^{3} c-2 a^{2} b c\right)\left(T^{*}\right)^{3}+\left(2 a^{2} b c\right)\left(T^{*}\right)^{2} \\
& +2 a T^{*}-2 a:=\bar{M}_{11}
\end{aligned}
$$

$$
\begin{aligned}
\hat{M}_{12}= & 1-(1-2 a) T^{*}:=\frac{\bar{M}_{12}}{m_{1}} \\
m_{1} \hat{M}_{21}= & \left(-2 a^{3} c+2 a^{2} b c\right)\left(T^{*}\right)^{3} \\
& +\left(-2 a^{2} b c-2 a^{2} c\right)\left(T^{*}\right)^{2}-2 a T^{*}+2 a:=\bar{M}_{21} \\
m_{1} \hat{M}_{22}= & \left(-2 a^{3} c+2 a^{2} b c+a^{2} c-a b c\right)\left(T^{*}\right)^{3} \\
& +\left(-2 a^{2} b c-3 a^{2} c+2 a b c+2 a c-b c\right)\left(T^{*}\right)^{2} \\
& +(-a b c-2 a c+2 b c) T^{*}-b c:=\bar{M}_{22},
\end{aligned}
$$

where $m_{1}=a c T^{*}\left(a T^{*}+b\left(1-T^{*}\right)\right)>0$. Hence,

$$
\begin{aligned}
m_{1} \operatorname{tr}(\hat{M})= & \left(a^{2} c-a b c\right)\left(T^{*}\right)^{3} \\
& +\left(-3 a^{2} c+2 a b c+2 a c-b c\right)\left(T^{*}\right)^{2} \\
& +(-a b c-2 a c+2 b c+2 a) T^{*}-2 a-b c \\
= & a^{2} c\left(T^{*}\right)^{2}\left(T^{*}-1\right)-2 a^{2} c\left(T^{*}\right)^{2} \\
& -a b c T^{*}\left(T^{*}-1\right)^{2}+2 a c T^{*}\left(T^{*}-1\right) \\
& -b c\left(T^{*}-1\right)^{2}+2 a\left(T^{*}-1\right)
\end{aligned}
$$

and so, $\operatorname{tr}(\hat{\boldsymbol{M}})<0$. Furthermore, $m_{1}^{2} \operatorname{det}(\hat{\boldsymbol{M}})=\operatorname{det}(\overline{\boldsymbol{M}})$, where $\overline{\boldsymbol{M}}=\left[\overline{\boldsymbol{M}}_{i j}\right]_{1 \leq i, j \leq 2}$. Using a row operation on $\overline{\boldsymbol{M}}$ and some laborious calculations, we conclude that $\operatorname{det}(\hat{\boldsymbol{M}})=2$ $\left(T^{*}-1\right) \alpha^{\prime}\left(T^{*}\right)$, which implies that $\operatorname{det}(\hat{\boldsymbol{M}})$ and $\alpha^{\prime}\left(T^{*}\right)$ have opposite signs. The assertion of Theorem (iii) now follows from the fact that the monotonicity of $\tilde{\alpha}(T)$ is opposite to that of $\tilde{S}$. We have just completed the proof of Theorem 2.2(iii). The proof of Theorem 2.2 is now complete.

${ }^{1} \mathrm{H}$. W. Hethcote, "The mathematics of infectious diseases," SIAM Rev. 42, 599-653 (2000).

${ }^{2}$ A. Barrat, M. Barthélemy, and A. Vespignani, Dynamical Processes on Complex Networks (Cambridge University Press, New York, 2008).

${ }^{3}$ M. E. J. Newman, "The structure and function of complex networks," SIAM Rev. 45, 167-256 (2003).

${ }^{4}$ V. Colizza, A. Barrat, M. Barthélemy, and A. Vespignani, "The role of the airline transportation network in the prediction and predictability of global epidemics," Proc. Natl. Acad. Sci. U. S. A. 103, 2015-2020 (2006).

${ }^{5}$ W. O. Kermack and A. G. McKendrick, "A contribution to the mathematical theory of epidemics," Proc. R. Soc. London A 115, 700-721 (1927).

${ }^{6}$ R. M. Anderson and R. M. May, Infectious Diseases of Humans: Dynamics and Control (Oxford University Press, Oxford, 1992).

${ }^{7}$ R. Pastor-Satorras and A. Vespignani, "Epidemic spreading in scale-free networks," Phys. Rev. Lett. 86, 3200-3203 (2001).

${ }^{8}$ R. Pastor-Satorras and A. Vespignani, "Epidemic dynamics and endemic states in complex networks," Phys. Rev. E 63, 066117 (2001).

${ }^{9}$ Y. Moreno, R. Pastor-Satorras, and A. Vespignani, "Epidemic outbreaks in complex heterogeneous networks," Eur. Phys. J. B 26, 521-529 (2002).

${ }^{10}$ M. E. J. Newman, "Spread of epidemic disease on networks," Phys. Rev. E 66, 016128 (2002).

${ }^{11}$ M. E. J. Newman, "Threshold effects for two pathogens spreading on a network," Phys. Rev. Lett. 95, 108701 (2005).

${ }^{12}$ Y.-Y. Ahn, H. Jeong, N. Masuda, and J. D. Noh, "Epidemic dynamics of two species of interacting particles on scale-free networks," Phys. Rev. E 74, 066113 (2006).

${ }^{13}$ S. Funk and V. A. A. Jansen, "Interacting epidemics on overlay networks," Phys. Rev. E 81, 036118 (2010).

${ }^{14}$ V. Marceau, P.-A. Noël, L. Hébert-Dufresne, A. Allard, and L. J. Dubé, "Modeling the dynamical interaction between epidemics on overlay networks," Phys. Rev. E 84, 026105 (2011).

${ }^{15}$ B. Karrer and M. E. J. Newman, "Competing epidemics on complex networks," Phys. Rev. E 84, 036106 (2011).

${ }^{16}$ C. Poletto, S. Meloni, V. Colizza, Y. Moreno, and A. Vespignani, "Host mobility drives pathogen competition in spatially structured populations," PLoS Comput. Biol. 9, e1003169 (2013). 
${ }^{17}$ F. D. Sahneh and C. Scoglio, "Competitive epidemic spreading over arbitrary multilayer networks," Phys. Rev. E 89, 062817 (2014).

${ }^{18}$ M. J. Alter, "Epidemiology of viral hepatitis and HIV co-infection," J. Hepatol. 44, S6-S9 (2006).

${ }^{19}$ L. J. Abu-Raddad, P. Patnaik, and J. G. Kublin, "Dual infection with HIV and malaria fuels the spread of both diseases in sub-Saharan Africa," Science 314, 1603-1606 (2006).

${ }^{20}$ A. Pawlowski, M. Jansson, M. Sköld, M. E. Rottenberg, and G. Källenius, "Tuberculosis and HIV co-infection," PLoS Pathog. 8, e1002464 (2012).

${ }^{21}$ World Health Organization, see http://apps.who.int/iris/bitstream/10665/ 137094/1/9789241564809_eng.pdf for Global tuberculosis report 2014 (2014).

${ }^{22}$ L. Chen, F. Ghanbarnejad, W. Cai, and P. Grassberger, "Outbreaks of coinfections: The critical role of cooperativity,” Europhys. Lett. 104, 50001 (2013).
${ }^{23}$ J. Sanz, C.-Y. Xia, S. Meloni, and Y. Moreno, "Dynamics of interacting diseases," Phys. Rev. X 4, 041005 (2014).

${ }^{24}$ W. Cai, L. Chen, F. Ghanbarnejad, and P. Grassberger, "Avalanche outbreaks emerging in cooperative contagions," Nat. Phys. 11, 936-940 (2015).

${ }^{25} \mathrm{P}$. Grassberger, L. Chen, F. Ghanbarnejad, and W. Cai, "Phase transitions in cooperative coinfections: Simulation results for networks and lattices," Phys. Rev. E 93, 042316 (2016).

${ }^{26}$ L. Chen, F. Ghanbarnejad, and D. Brockmann, "Fundamental properties of cooperative contagion processes," New J. Phys. 19, 103041 (2017).

${ }^{27}$ J. P. Rodríguez, F. Ghanbarnejad, and V. M. Eguíluz, "Risk of coinfection outbreaks in temporal networks: A case study of a hospital contact network," Front. Phys. 5, 46 (2017). 